\title{
A formulation of convective entrainment in terms of mixing efficiency
}

\author{
C. CHEMEL† AND C. STAQUET \\ Laboratoire des Ecoulements Géophysiques et Industriels, CNRS / UJF / INPG \\ BP 53, 38041 Grenoble Cedex 9, France
}

(Received 7 August 2006 and in revised form 4 March 2007)

The entrainment across a stably stratified interface forced by convective motions is discussed in the light of the mixing efficiency of the entrainment process. The context is the convectively driven atmospheric boundary layer and we focus on the regime of equilibrium entrainment, i.e. when the boundary-layer evolution is in a quasi-steady state. The entrainment law is classically based on the ratio $\mathscr{R}$ of the negative of the heat flux at the interface to the heat flux at the ground surface. We propose a parameterization for $\mathscr{R}$ that involves the mixing efficiency and the thickness of the interface, which matches well the direct computation of $\mathscr{R}$ from a high-resolution large-eddy simulation. This result enables us to derive modified expressions for the classical entrainment laws (the so-called zero- and first-order models) as a function of the mixing efficiency. We show that, when the thickness of the interface is ignored (zero-order model), the scaling factor $\mathscr{A}$ in the entrainment law is the flux Richardson number. This parameterization of $\mathscr{A}$ is further improved when the thickness of the interface is considered (first-order model), as shown by the direct computation of $\mathscr{A}$ from the large-eddy simulation.

\section{Introduction}

The interfacial layer (IL) between the convectively driven atmospheric boundary layer and the stably stratified free atmosphere above is subjected to intense turbulent mixing, due to entrainment of air by convective motions. As a result, the IL rises or, equivalently, the boundary layer deepens. As pointed out for instance by Otte \& Wyngaard (2001), the modelling of the entrainment process is an essential issue for both oceanic and atmospheric applications. Thus, several entrainment law formulations have been derived to date, which relate the entrainment velocity, namely the velocity at which the interface rises, to measurable parameters of the mixed and interfacial layers.

In the zero-order model (ZOM) proposed by Lilly (1968), the thickness of the IL is assumed infinitesimal, and the temperature profile exhibits a jump across that interface (see figure $1 a$ ). The IL altitude, denoted by $z_{i}$, is defined as the level where the heat flux is minimum (being negative). Herein we consider a horizontally homogeneous boundary layer without large-scale subsidence. Let $\Theta$ and $\mathscr{F}$ denote the horizontally averaged virtual potential temperature and heat flux, respectively. Lilly (1968) showed

$\dagger$ Present address: Centre for Atmospheric and Instrumentation Research, University of Hertfordshire, College Lane Campus, Hatfield, Herts AL10 9AB, UK. Author to whom correspondence should be addressed: c.chemel@herts.ac.uk. 

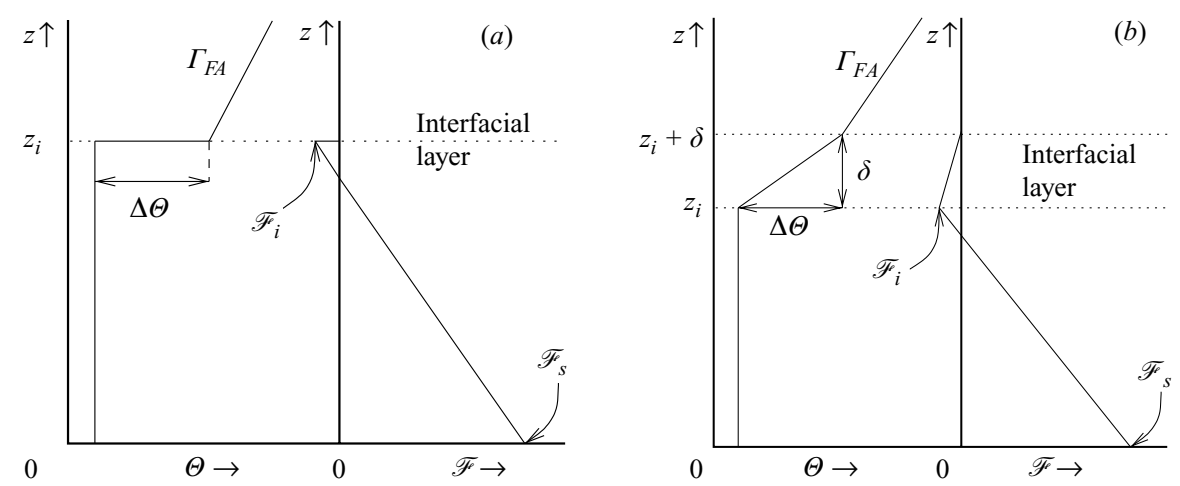

FIgURE 1. Profiles of typical virtual potential temperature $\Theta$ and heat flux $\mathscr{F}$ for the convectively driven boundary layer. (a) Zero-order model (ZOM) by Lilly (1968); $(b)$ first-order model (FOM) by Betts (1974). The strength of the capping inversion is denoted by $\Delta \Theta$. The interfacial-layer thickness is designated $\delta$ and $\Gamma_{F A}$ is the $\Theta$ lapse rate in the free atmosphere.

that the entrainment velocity, $w_{e} \equiv \dot{z}_{i}$, can be expressed as

$$
w_{e}=-\mathscr{F}_{i} / \Delta \Theta
$$

where $\mathscr{F}_{i}$ is the heat flux at the interface and $\Delta \Theta$ is the strength of the capping inversion.

The difficulty, once $\Delta \Theta$ is known, is to estimate the entrainment heat flux $\mathscr{F}_{i}$ and a simple closure was proposed by assuming that $\mathscr{F}_{i}$ is a fixed fraction of the surface heat flux $\mathscr{F}_{s}$, i.e. the flux ratio,

$$
\mathscr{R} \equiv-\mathscr{F}_{i} / \mathscr{F}_{s},
$$

has a constant value. This value is commonly taken as 0.2 (e.g. Stull 1976). Equations (1.1) and (1.2) together yield a simple model for the entrainment velocity,

$$
w_{e}=\mathscr{R} \frac{\mathscr{F}_{s}}{\Delta \Theta} .
$$

This expression for $w_{e}$ may be written in a non-dimensional form by introducing a convective velocity, $w_{\star} \equiv\left(g \beta \mathscr{F}_{s} z_{i}\right)^{1 / 3}$, with $g$ being the gravitational acceleration and $\beta$ the coefficient of thermal expansion, and a bulk (overall) Richardson number,

$$
R i_{B} \equiv \frac{g \beta \Delta \Theta z_{i}}{w_{\star}^{2}}
$$

This yields the classical ZOM formulation of the entrainment law,

$$
w_{e} / w_{\star}=\mathscr{A}_{0}^{\mathscr{R}} R i_{B}^{-1},
$$

with $\mathscr{A}_{0}^{\mathscr{R}}=\mathscr{R}$. Hereafter the subscript 0 refers to the ZOM framework.

In first-order models (FOMs), the finite thickness $\delta$ of the IL is taken into account (e.g. Betts 1974; van Zanten et al. 1999). Betts (1974) assumed that the mixed layer extends up to $z_{i}$, and that above $z_{i}$ the heat flux increases linearly to zero at $z_{i}+\delta$ (see figure 1b). Assuming that the IL thickness is constant, Betts (1974) showed that the entrainment velocity can be written as

$$
w_{e}=\frac{-\mathscr{F}_{i}+\delta \partial \Theta^{\star} / \partial t}{\Delta \Theta},
$$

where $\Theta^{\star}=\left[\Theta\left(z_{i}\right)+\Theta\left(z_{i}+\delta\right)\right] / 2$. In the limit of infinitely small thickness of the interface $(\delta \rightarrow 0)$, equation (1.6) reduces to equation (1.1), as it should. Note that rigorously we should use another notation for $\Delta \Theta$ (and consequently $R i_{B}$ ) within the FOM framework (see the discussion in $\S 4$ ). Using the definitions of the convective 
velocity and the bulk Richardson number above, equation (1.6) can be rewritten as

$$
w_{e} / w_{\star}=\mathscr{A}_{1}^{\mathscr{R}} R i_{B}^{-1},
$$

where $\mathscr{A}_{1}^{\mathscr{R}}=\mathscr{R}+\left(\delta / \mathscr{\mathscr { F }}_{s}\right) \partial \Theta^{\star} / \partial t$. This is the classical expression of the entrainment law in the FOM framework. Note that the subscript 1 refers to the order of the model.

To account for the smooth vertical profiles of both $\Theta$ and $\mathscr{F}$ in the real atmosphere, more general formulations have been derived from integral parameters of the IL (e.g. Fedorovich \& Mironov 1995). General-structure models thus obtained are more realistic but contain empirical dimensionless functions, which need to be carefully estimated from either field or laboratory experiments or numerical simulations.

From an experimental point of view, the entrainment processes across a density interface have been studied extensively (see Hopfinger 1987; Fernando 1991, for reviews, as well as references therein). When entrainment results from grid-generated turbulence, the dimensionless entrainment velocity, $w_{e} / w_{\star}$, was found to vary as $R i_{B}^{-n}$, where $n$ is in the range 1-2 depending upon the values of the Prandtl and Richardson numbers. For strong enough stratification of the interface, Turner (1968) found that for density differences produced by heat alone $n$ is close to 1 , and in the presence of a salinity difference across the interface $n$ is close to $3 / 2$. In the convection tank experiment of Deardorff, Willis \& Stockton (1980), the dimensionless entrainment velocity was found to vary as

$$
w_{e} / w_{\star}=\mathscr{A} R i_{B}^{-1},
$$

with $\mathscr{A} \simeq 0.25$. As noted by Deardorff et al. (1980), this is equation (1.5) with $\mathscr{A}=\mathscr{R}$. But these authors found that their data could also be fitted by a $R i_{B}^{-3 / 2}$ power law, so that there seemed to be no clear conclusion on the values of both $n$ and $\mathscr{A}$.

The entrainment-law formulation has also been extensively investigated from large-eddy simulations (LESs) of the convective atmospheric boundary layer. The entrainment law (1.8) is generally confirmed with $\mathscr{A} \simeq 0.25$ in the regime of equilibrium entrainment, which is realized when the turbulent regime in the mixed layer is in a quasi-steady state (e.g. Sullivan et al. 1998; Fedorovich, Conzemius \& Mironov 2004; Chemel, Staquet \& Chollet 2007). Nevertheless, LES investigations in the literature show a scatter of about $30 \%$ in the value of $\mathscr{A}$ (e.g. Stevens \& Lenschow 2001). Stevens et al. (2000) also noted that the entrainment velocity is clearly dependent on numerical and subgrid-scale mixing, as well as on the details of the numerics. This result indicates that only high-resolution LESs would provide reliable estimates of $\mathscr{A}$ since, as the resolution is increased, these dependences should decrease.

The main purpose of the present paper is to investigate the link between the quantification of mixing at the interface and the entrainment law. To proceed, we apply the concept of mixing efficiency to both the ZOM and FOM frameworks, and use a high-resolution LES of the convectively driven boundary layer to assess the validity of our approach.

\section{Energy considerations}

\subsection{Basic concepts of mixing}

When mixing occurs in a stably stratified fluid, the centre of mass of the fluid system rises. Hence, the potential energy of the fluid system increases through mixing. The term potential energy should be specified. One should distinguish the potential energy associated with reversible processes, like internal gravity wave motions, from the potential energy associated with irreversible processes, like mixing. The former is the available potential energy (Lorenz 1955) and vanishes when the flow returns to its 
rest state. Conversely, the latter is the minimum potential energy of the fluid system (usually referred to as the background potential energy, denoted by $E_{b}$ ) and always increases through mixing.

The amount of energy brought into a fluid system, by a paddle in a laboratory experiment (in which case the energy input is kinetic) or by heating from below as in the present case (in which case the energy input is potential), is thus dissipated via two sinks: (i) kinetic energy dissipation and (ii) the mixing of the active scalar (the virtual potential temperature in the present case), which locally reduces its gradient.

Mixing in a stably stratified fluid is most usefully quantified by the mixing efficiency (e.g. Osborn \& Cox 1972). The mixing efficiency, denoted by $\gamma$, is defined as the ratio of the increase in the background potential energy per time unit, $\dot{E}_{b}$, to the dissipation rate of turbulent kinetic energy $\mathscr{E}$ :

$$
\gamma \equiv \dot{E}_{b} / \mathscr{E},
$$

where $E_{b}$ and $\mathscr{E}$ are volume-averaged quantities. This quantification of mixing is equivalent to that of the flux Richardson number $R i_{f}$ as obtained by Linden (1979) in forced turbulence laboratory experiments. In that case, $\dot{E}_{b}$ is instead compared to the input rate of energy to the fluid system per time unit, denoted by $\dot{\mathscr{M}}$, yielding

$$
R i_{f} \equiv \dot{E}_{b} / \dot{\mathscr{M}} .
$$

When a statistically steady regime is reached, $\dot{\mathscr{M}}=\dot{E}_{b}+\mathscr{E}$, implying that the flux Richardson number and the mixing efficiency are simply related by

$$
R i_{f}=\frac{\gamma}{\gamma+1} .
$$

Note that $R i_{f}$ is the same as the parameter $R^{\star}$ introduced by Manins \& Turner (1978).

Let us assume that the turbulent regime in the mixed layer is in a statistically steady state. This is a valid assumption if the entrainment velocity $w_{e}$ is much smaller than the typical velocity of the convective motions $w_{\star}$. Hence we assume that $w_{e} \ll w_{\star}$, which is the case in the convectively driven atmospheric boundary layer.

\subsection{Application to the zero-order model}

Let us start from the heat balance equation, $\partial \Theta / \partial t=-\partial \mathscr{F} / \partial z$, in the Boussinesq approximation, neglecting subsidence and molecular effects. In the mixed layer, $\Theta$ is constant with height, so that the heat flux $\mathscr{F}$ decreases linearly with height (see figure $1 a)$ :

$$
\mathscr{F}=\mathscr{F}_{s}\left[\left(1-\frac{z}{z_{i}}\right)-\mathscr{R} \frac{z}{z_{i}}\right] .
$$

As is usual, we consider the equation for the turbulent kinetic energy averaged over the mixed-layer depth (e.g. Stull 1976). For strong enough stability of the air above and neglecting the flux due to upward radiation of energy from the top of the boundary layer, this equation may be written as

$$
g \beta \frac{1}{z_{i}} \int_{0}^{z_{i}} \mathscr{F} \mathrm{d} z=\frac{1}{z_{i}} \int_{0}^{z_{i}} \bar{\varepsilon} \mathrm{d} z,
$$

where $\bar{\varepsilon}$ is the horizontally averaged dissipation rate of kinetic energy.

We shall now partition the buoyancy flux averaged over the mixed layer into positive and negative contributions, which are identified as energy production and mixing terms, respectively. We adopt the process-partitioning originally proposed by 

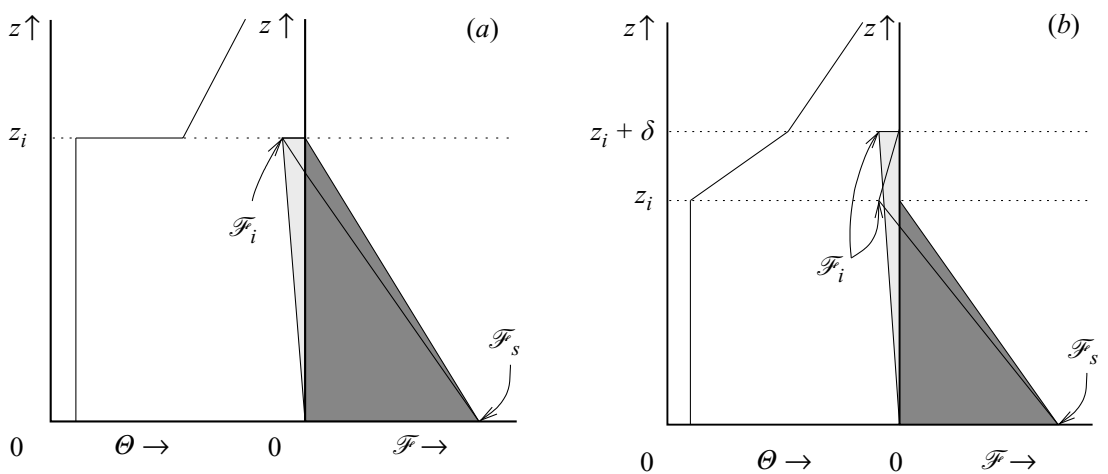

FiguRE 2. Partitioning to retrieve the energy production part (dark grey) and the mixing part (light grey) of the buoyancy flux. (a) Zero-order model (ZOM) by Lilly (1968); $(b)$ first-order model (FOM) by Betts (1974).

Manins \& Turner (1978) (see also Zilitinkevich 1991; Fedorovich et al. 2004). From equation (2.4), the buoyancy flux profile averaged over the mixed layer,

$$
g \beta \frac{1}{z_{i}} \int_{0}^{z_{i}} \mathscr{F}^{\mathrm{d} z}=\frac{1}{2} g \beta \mathscr{F}_{s}+\frac{1}{2} g \beta \mathscr{F}_{i},
$$

is thus the sum of a positive contribution due to ground surface heating, $g \beta \mathscr{F}_{s} / 2$, which we identify as $\dot{M}$, and a negative contribution due to convective entrainment, $g \beta \mathscr{F}_{i} / 2$, which we identify as $-\dot{E}_{b}$; the integration extends all over the mixed-layer depth as we may expect from updraughts and downdraughts. Hence, $\dot{\mathscr{M}}=g \beta \mathscr{F}_{s} / 2$ is the energy production due to ground surface heating (displayed in dark grey in figure $2 a$ ), and $-\dot{E}_{b}=g \beta \mathscr{F}_{i} / 2$ is the fraction of buoyancy production used on entrainment (displayed in light grey in figure $2 a$ ). Note that this expression for $\dot{E}_{b}$ can be derived easily by computing the potential energy averaged over the mixedlayer depth, taking the time derivative, and using the heat balance equation (see Winters \& D'Asaro 1996, equation (15)). It follows that the flux ratio is the flux Richardson number $R i_{f}$, i.e. in terms of mixing efficiency,

$$
\mathscr{R}_{0}^{\gamma}=\frac{\gamma}{\gamma+1}
$$

where the superscript $\gamma$ denotes a result based on mixing efficiency. This means that a fraction $\gamma /(\gamma+1)$ of the energy made available by ground surface heating is used to deepen the mixed layer (or equivalently to lift the interface). This result was guessed by several authors in the sense that the flux ratio $\mathscr{R}$ was referred to as the energy efficiency (e.g. Lilly 2002, p. 3346), the entrainment efficiency (e.g. Caldwell, Bretherton \& Wood 2005, p. 3787), or the general entrainment flux ratio (e.g. Conzemius \& Fedorovich 2006, p. 1158).

The entrainment law (1.5) may thus be expressed as a function of the mixing efficiency $\gamma$ using equation (2.7), namely

$$
w_{e} / w_{\star}=\mathscr{A}_{0}^{\gamma} R i_{B}^{-1},
$$

with $\mathscr{A}_{0}^{\gamma}=\gamma /(\gamma+1)$. At this point, we recall that this equation is valid when $w_{e} / w_{\star} \ll 1$, that is, since $\gamma<1$, when $R i_{B} \gg 1$. Hence, the interface should be strongly stratified for this analysis to apply, as already assumed for the air above. 


\subsection{Application to the first-order model}

For the FOM, both $\Theta$ and $\mathscr{F}$ are made up of linear segments (see figure $1 b$ ). The expression for the heat flux $\mathscr{F}$ is straightforward: for $0 \leqslant z \leqslant z_{i}, \mathscr{F}$ is expressed by equation (2.4) while for $z_{i} \leqslant z \leqslant z_{i}+\delta, \mathscr{F}=\left(\mathscr{R} \mathscr{F}_{s} / \delta\right)\left[z-\left(z_{i}+\delta\right)\right]$. As in $\S 2.2$, we partition the buoyancy flux averaged from the ground surface up to the free atmosphere,

$$
g \beta \frac{1}{z_{i}+\delta} \int_{0}^{z_{i}+\delta} \mathscr{F} \mathrm{d} z=\frac{1}{2} g \beta \frac{z_{i}}{z_{i}+\delta} \mathscr{F}_{s}+\frac{1}{2} g \beta \mathscr{F}_{i},
$$

into a positive contribution, $\dot{M}$, and a negative contribution, $-\dot{E}_{b}$. Consistent with $\S 2.2$, we shall assume that the positive contribution due to ground surface heating occurs through updraughts that extend up to the top of the mixed layer $z_{i}$. This implies that $\dot{\mathscr{M}}=g \beta\left[z_{i} /\left(z_{i}+\delta\right)\right] \mathscr{F}_{s} / 2$, which implies that $\dot{E}_{b}=-g \beta \mathscr{F}_{i} / 2$. Both the positive and negative contributions to the averaged buoyancy flux are illustrated in figure $2(b)$. It follows that, in this FOM framework, the flux ratio and the mixing efficiency $\gamma$ are related by

$$
\mathscr{R}_{1}^{\gamma}=\frac{z_{i}}{z_{i}+\delta} \frac{\gamma}{\gamma+1} .
$$

Assuming that the IL thickness is constant in the regime of equilibrium entrainment and using the FOM by Betts (1974), equation (2.10) for the flux ratio enables us to derive a modified expression of the entrainment law (1.7) as a function of $\gamma$, namely

$$
w_{e} / w_{\star}=\mathscr{A}_{1}^{\gamma} R i_{B}^{-1},
$$

where $\mathscr{A}_{1}^{\gamma}=\left[z_{i} /\left(z_{i}+\delta\right)\right] \gamma /(\gamma+1)+\left(\delta / \mathscr{F}_{s}\right) \partial \Theta^{\star} / \partial t$.

\section{Computation of the mixing efficiency}

In this section, we compute directly the mixing efficiency from the high-resolution LES data of Chemel et al. (2007) in the regime of equilibrium entrainment. A detailed description of the LES is given in Chemel et al. (2007). The horizontal domain is $5.12 \mathrm{~km} \times 5.12 \mathrm{~km}$ and the domain height is $4.535 \mathrm{~km}$. The computation was performed with the ARPS (Advanced Regional Prediction System) model (Xue, Droegemeier \& Wong 2000), using a horizontal resolution of $20 \mathrm{~m}$. The vertical resolution was $20 \mathrm{~m}$ over the mixed-layer depth, $5 \mathrm{~m}$ within the IL and $50 \mathrm{~m}$ above. The model was initialized using a 0900 EST (local time) sounding of Day 33 of the Wangara experiment (Clarke et al. 1971). In the present study, we use the data from 1200 EST to $1330 \mathrm{EST}$, for which the boundary-layer evolution is in a quasi-steady state and the IL is strongly stratified $\left(R i_{B}>10\right)$.

Here, the attention is focused on the IL. We thus consider a volume $\mathscr{V}$ extending on both sides of the interface, from a level above the ground surface (at $\left.z / z_{i}=0.5\right)$ up to a level far above the upper boundary of the IL (at $z / z_{i}=1.5$ ). These boundaries may be regarded as geometric boundaries, which do not interfere with the IL. The temporal evolution of $E_{b}$ satisfies the equation (Winters \& D'Asaro 1996)

$$
\dot{E}_{b}=\Phi_{d}+\mathscr{F}_{\text {surf }}^{a d v}+\mathscr{F}_{\text {surf }}^{\text {dif }} \text {, }
$$

where all four terms are averaged over $\mathscr{V} . \Phi_{d}$ is the rate of change of $E_{b}$ due to mixing across the surfaces of constant resolved (filtered) virtual potential temperature $\underset{\Theta}{\widetilde{\Theta}} ; \mathscr{F}_{\text {surf }}^{\text {sadv }}$ and $\mathscr{F}_{\text {surf }}^{\text {dif }}$ are the rate of change of $E_{b}$ due to advection and diffusion of $\Theta$ across the boundaries of $\mathscr{V}$. The lateral boundaries of our LES being periodic, there is no net transport into or out of the computational domain through those 


$$
\begin{array}{llc} 
& \multicolumn{2}{c}{\text { Model framework }} \\
\cline { 2 - 3 } \mathscr{R} \equiv-\frac{\mathscr{F}_{i}}{\mathscr{F}_{s}} & \mathscr{R}_{0}^{\gamma}=\frac{\gamma}{\gamma+1} & \mathscr{R}_{1}^{\gamma}=\frac{z_{i}}{z_{i}+\delta} \frac{\gamma}{\gamma+1} \\
& \mathscr{A}_{0}^{\mathscr{R}}=\mathscr{R} & \mathscr{A}_{1}^{\mathscr{R}}=\mathscr{R}+\frac{\delta}{\mathscr{F}_{s}} \frac{\partial \Theta^{\star}}{\partial t} \\
\mathscr{A} \equiv \frac{w_{e}}{w_{\star}} R i_{B} & \mathscr{A}_{0}^{\gamma}=\frac{\gamma}{\gamma+1} & \mathscr{A}_{1}^{\gamma}=\frac{z_{i}}{z_{i}+\delta} \frac{\gamma}{\gamma+1}+\frac{\delta}{\mathscr{F}_{s}} \frac{\partial \Theta^{\star}}{\partial t}
\end{array}
$$

TABLE 1. Definition (first column) and parameterization of the flux ratio $\mathscr{R}$ and of the scaling factor $\mathscr{A}$ in the entrainment law, using the zero-order model (ZOM) by Lilly (1968) (second column) and the first-order model (FOM) by Betts (1974) (third column). All parameters are defined in the text.

boundaries. At the upper boundary where the fluid is at rest, $\mathscr{F}_{\text {surf }}^{a d v}$ vanishes and $\mathscr{F}_{\text {surf }}$ may be neglected. Across the lower boundary both fluxes are zero since the fluid is well mixed there.

Thus, the change of $E_{b}$ occurs only through mixing, so that only the location where mixing occurs, namely the IL, contributes to this change. The term $\Phi_{d}$ is computed as a volume average over $\mathscr{V}$ of the local diffusive flux across the $\widetilde{\Theta}$ surfaces, denoted by $\varphi_{d}$, multiplied by $g \beta$. Winters \& D'Asaro (1996) derived an exact expression for $\varphi_{d}$ :

$$
\varphi_{d}=\frac{\left\langle\kappa_{t}|\nabla \widetilde{\Theta}|^{2}\right\rangle}{\mathrm{d} \widetilde{\Theta}_{s} / \mathrm{d} z_{\star}},
$$

where $\kappa_{t}$ is the subgrid-scale thermal diffusivity. The $\langle\square\rangle$ operator denotes an average along a constant $-\widetilde{\Theta}$ surface. $\widetilde{\Theta}_{s}\left(z_{\star}\right)$ is the stable $\widetilde{\Theta}$ profile obtained by an adiabatic rearrangement of the fluid particles (elementary volumes of side equal to the minimum grid size). From a practical point of view, the fluid particles are moved to their new vertical position $z_{\star}$ according to the value of their virtual potential temperature, the lowest particle being the heaviest. In a three-dimensional fluid volume, a similar methodology is applied. The stably stratified profile is constructed by filling the successive horizontal planes with the ordered particles. Then, the background $\widetilde{\Theta}_{s}$ profile at each plane is computed by a horizontal average. The mixing efficiency $\gamma$ at the interface is simply inferred from equations (2.1), (3.1) and (3.2), $\mathscr{E}$ being computed from the subgrid-scale model. Note that $\gamma$ accounts for the IL thickness since it is based on the sorted profile $\widetilde{\Theta}_{s}\left(z_{\star}\right)$.

The mixing efficiency was computed every $30 \mathrm{~min}$ from 1200 EST to 1330 EST. All values were between 0.26 and 0.30 (being equal, as time elapses, to $0.28,0.30$, 0.29 and 0.26 , respectively). This range is consistent with the one found in turbulent stably stratified flows, whether in the ocean (e.g. Moum \& Caldwell 1995), in the stratosphere (e.g. Wilson, Dalaudier \& Bertin 2005), in laboratory experiments (e.g. Linden 1979) or in numerical simulations (e.g. Staquet 2000).

\section{Evaluation of the models for $\mathscr{R}$ and $\mathscr{A}$ based on $\gamma$}

Our purpose in this section is to compare the numerical value of $\mathscr{R}$, as computed from the expressions based on $\gamma$ within the ZOM and FOM frameworks, with that computed directly from its definition (1.2). These expressions are recalled in table 1. 

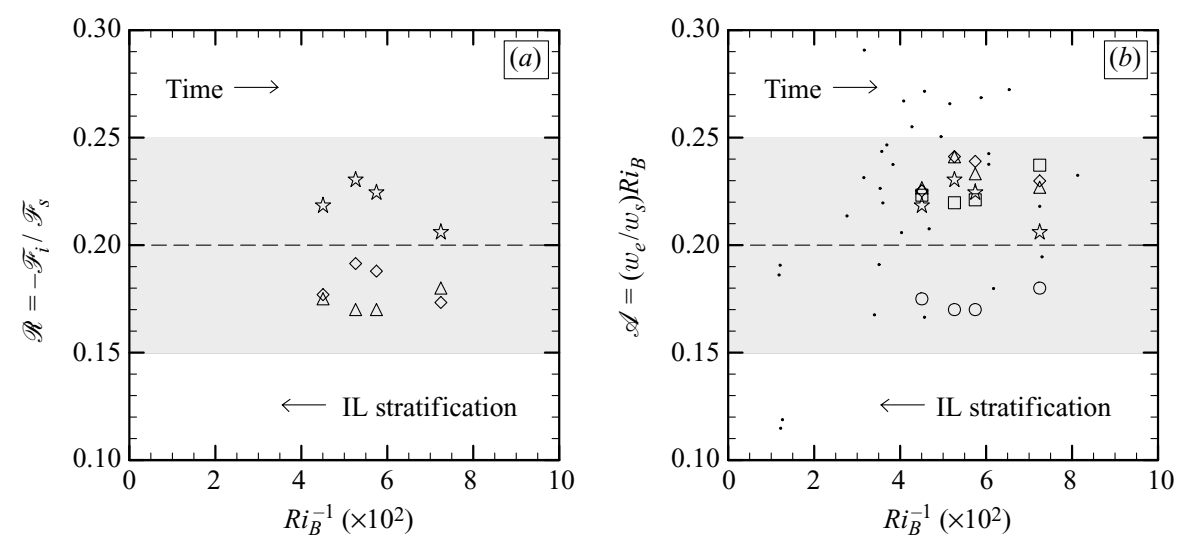

FIGURE 3. (a) Flux ratio computed from the LES data of Chemel et al. (2007) versus $R i_{B}^{-1}$ : $\triangle, \mathscr{R} ; i, \mathscr{R}_{\star}^{\gamma} ; \diamond, \mathscr{R}_{1}^{\gamma}$. (b) Dimensionless parameter $\mathscr{A}=\left(w_{e} / w_{\star}\right) R i_{B}$ computed from the LES data, as a function of $R i_{B}^{-1}: \triangle, \mathscr{A}_{\text {num }} ;$ is, $\mathscr{A}_{\star}^{\gamma} ; \diamond, \mathscr{A}_{1}^{\gamma} ; O, \mathscr{A}_{0}^{\mathscr{R}} ; \square, \mathscr{A}_{1}^{\mathscr{R}}$. Experimental values from the convection tank measurements of Deardorff et al. (1980) are also included as $\cdot$. The shaded area represents $\mathscr{R}$ and $\mathscr{A}$ in the range $0.15-0.25$. All expressions for $\mathscr{R}$ and $\mathscr{A}$ are given in table 1 , while $R i_{B}$ is defined by equation (1.4).

We also compare the numerical values of $\mathscr{A}$ as computed from the four expressions derived, which are recalled in table 1, with that computed directly from its definition (1.8), denoted by $\mathscr{A}_{\text {num }}$, and with the experimental measurements of Deardorff et al. (1980). These comparisons are displayed in figure $3(a)$ for $\mathscr{R}$ and in figure $3(b)$ for $\mathscr{A}$.

The retrieval of the LES data needs to be carefully discussed as it should be consistent with the model framework. The retrieval procedure we used is as detailed by Fedorovich et al. (2004, p. 287), except for $\Delta \Theta$ in the ZOM framework. As their procedure proved to be difficult to apply (because the buoyancy frequency in the free atmosphere is not constant in our case), we kept the same value of $\Delta \Theta$ in both the ZOM and FOM frameworks, i.e. that of the temperature jump $\Theta\left(z_{i}+\delta\right)-\Theta\left(z_{i}\right)$. Note that $\Delta \Theta$ appears only in the definition of the bulk Richardson number $R i_{B}$, so that $R i_{B}$ has the same value for either model, which makes comparisons easier. For the mixing efficiency, we have already noted that the computation of $\gamma$ involves the IL thickness. Hence, the retrieval of the parameters $\mathscr{R}_{0}^{\gamma}$ and $\mathscr{A}_{0}^{\gamma}$ from the LES is not fully consistent with the ZOM framework. In figure 3 , we shall thus refer to the computed values of these parameters as $\mathscr{R}_{\star}^{\gamma}$ and $\mathscr{A}_{\star}^{\gamma}$, respectively, in order to avoid confusion with the notation $\mathscr{R}_{0}^{\gamma}$ and $\mathscr{A}_{0}^{\gamma}$, which would imply that $\gamma$ has been computed for an infinitely small thickness of the IL.

The LES computed values for $\mathscr{R}$ in figure $3(a)$ are between 0.17 and 0.18 , in agreement with the standard value of 0.2 taken for this ratio. The prediction by the FOM model is very good, the relative difference between $\mathscr{R}_{1}^{\gamma}$ and $\mathscr{R}$ being less than $10 \%$ on average. Conversely, $\mathscr{R}_{\star}^{\gamma}$ is a poor approximation of the flux ratio, the relative difference being between $14 \%$ and $36 \%$ as $R i_{B}$ varies. Figure $3(a)$ therefore shows that, when our LES data are considered, a reliable model for the flux ratio is provided by the expression $\left[z_{i} /\left(z_{i}+\delta\right)\right] \gamma /(\gamma+1)$, that is, assuming $\delta / z_{i}$ to be small, $\left(1-\delta / z_{i}\right) \gamma /(\gamma+1)$.

Figure $3(b)$ shows that $\mathscr{A}_{0}^{\mathscr{R}}$ (computed directly as $-\mathscr{F}_{i} / \mathscr{F}_{s}$ ) is not a good approximation of $\mathscr{A}_{\text {num }}$ whatever $R i_{B}$, as is well known (e.g. Sullivan et al. 1998; Fedorovich et al. 2004). The computation of $\mathscr{R}$ needs to be consistent with the ZOM 
framework. When $\mathscr{R}$ is approximated by $\gamma /(\gamma+1)$, better results are obtained. We note that the relative difference between $\mathscr{A}_{\star}^{\gamma}$ (namely $\gamma /(\gamma+1)$ ) and $\mathscr{A}_{\text {num }}$ is always less than $9 \%$ whatever $R i_{B}$. This result may be explained by recalling that the computation of $\gamma$ implicitly depends upon the finite thickness of the IL. However, the explicit introduction of the IL thickness does not improve significantly the modelling of $\mathscr{A}$ compared with $\mathscr{A}_{\star}^{\gamma}$ if the mixing efficiency is not taken into account as well: $\mathscr{A}_{1}^{\mathscr{R}}$ does not perform better than $\mathscr{A}_{\star}^{\gamma}$ but $\mathscr{A}_{1}^{\gamma}$ does remarkably. Indeed, the relative difference between $\mathscr{A}_{1}^{\gamma}$ and $\mathscr{A}_{\text {num }}$ is of a few percent whatever $R i_{B}$. Hence a good estimate of $\mathscr{A}$ is obtained by using the flux Richardson number and this estimate becomes excellent when the IL thickness is taken into account.

\section{Conclusions}

The objective of this work was to introduce the mixing efficiency into the parameterization of the entrainment process at the top of the convectively driven boundary layer. We focus upon the regime of equilibrium entrainment, when the Richardson number at the interface is high enough (say $R i_{B}>10$ ). The parameterization of the entrainment process usually relies on the ratio $\mathscr{R}$ of the negative of the heat flux at the interface to the heat flux at the ground surface, which is usually taken constant, with value 0.2 .

We proposed a parameterization for $\mathscr{R}$ based on the mixing efficiency and on the thickness of the IL. This is expression $\mathscr{R}_{1}^{\gamma}$ in table 1 , which can be further approximated by $\left(1-\delta / z_{i}\right) \gamma /(\gamma+1)$. With $\gamma \simeq 0.3$, which we computed directly from our LES data using the method proposed by Winters et al. (1995), and $\delta / z_{i} \simeq 0.2$ (also from the LES), this yields $\mathscr{R} \simeq 0.2$.

We next used the classical zero- and first-order model frameworks to derive new expressions for the entrainment law based on the mixing efficiency. Our purpose was to explain the value of about 0.2 generally obtained for the scaling factor $\mathscr{A}$ in the entrainment law. We showed that a good approximation of $\mathscr{A}$ is provided by the flux Richardson number of the entrainment process and that an excellent model is obtained when the IL thickness is further taken into account. This is expression $\mathscr{A}_{1}^{\gamma}$ in table 1.

From a practical point of view, the present parameterizations rely on the computation or measurement of $\gamma$. The direct method we used makes it possible to estimate $\gamma$ from LES data. Understanding the approximate 0.2 value found for $\gamma$ in stably stratified flows would provide final closure but this is still a challenge, despite attempts that have been made in specific contexts (e.g. McEwan 1983).

The authors thank two referees for their valuable comments, which led to substantial improvements to the manuscript.

\section{REFERENCES}

BetTs, A. K. 1974 Reply to comment on the paper 'non-precipitating cumulus convection and its parameterization'. Q. J. R. Met. Soc. 100, 469-471.

Caldwell, P., Bretherton, C. S. \& Wood, R. 2005 Mixed-layer budget analysis of the diurnal cycle of entrainment in Southeast Pacific stratocumulus. J. Atmos. Sci. 62, 3775-3791.

Chemel, C., Staquet, C. \& Chollet, J.-P. 2007 Eulerian- and Lagrangian-based estimates of convective entrainment rate from large-eddy simulation. J. Atmos. Sci. (submitted).

Clarke, R. H., Dyer, A. J., Brook, R. R., Reid, D. G. \& Troup, A. J. 1971 The Wangara experiment: Boundary layer data. Tech. Paper 19. CSIRO Atmospheric Research, Aspendale, Australia. 
Conzemius, R. \& Fedorovich, E. 2006 Dynamics of sheared convective boundary layer entrainment. Part I: methodological background and large-eddy simulations. J. Atmos. Sci. 63, 11511178.

Deardorff, J. W., Willis, G. E. \& Stockton, B. H. 1980 Laboratory studies of the entrainment zone of a convectively mixed layer. J. Fluid Mech. 100, 41-64.

Fedorovich, E., Conzemius, R. \& Mironov, D. 2004 Convective entrainment into a shear-free, linearly stratified atmosphere: bulk models reevaluated through large eddy simulations. J. Atmos. Sci. 61, 281-295.

Fedorovich, E. E. \& Mironov, D. V. 1995 A model for a shear-free convective boundary layer with parameterized capping inversion structure. J. Atmos. Sci. 52, 83-95.

Fernando, H. J. S. 1991 Turbulent mixing in stratified fluids. Anuu. Rev. Fluid Mech. 23, 455493.

Hopfinger, E. J. 1987 Turbulence in stratified fluids: a review. J. Geophys. Res. 92, 5287-5303.

LiLLY, D. K. 1968 Models of cloud-topped mixed layers under a strong inversion. Q. J. R. Met. Soc. 94, 292-309.

Lilly, D. K. 2002 Entrainment into mixed layers. Part I: sharp-edged and smoothed tops. J. Atmos. Sci. 59, 3340-3352.

Linden, P. F. 1979 Mixing in stratified fluids. Geophys. Astrophys. Fluid Dyn. 13, 3-23.

LORENZ, E. N. 1955 Available potential energy and the maintenance of the general circulation. Tellus 7, 157-167.

Manins, P. C. \& Turner, J. S. 1978 The relation between the flux ratio and energy ratio in convectively mixed layers. Q. J. R. Met. Soc. 104, 39-44.

McEwan, A. D. 1983 Internal mixing in stratified fluids. J. Fluid Mech. 128, 59-80.

Moum, J. N. \& Caldwell, D. R. 1995 Turbulence and mixing in the ocean. Rev. Geophys. 33, $1385-1394$.

Osborn, T. R. \& Cox, C. S. 1972 Oceanic fine structure. Geophys. Fluid Dyn. 3, 321-345.

OtTE, M. J. \& WyngaARD, J. C. 2001 Stably stratified interfacial-layer turbulence from large-eddy simulation. J. Atmos. Sci. 58, 3424-3442.

Staquet, C. 2000 Mixing in a stably stratified shear layer: two- and three-dimensional numerical experiments. Fluid Dyn. Res. 27, 367-404.

Stevens, B., Bell, J. B., Almgren, A. S., Beckner, V. E. \& Rendleman, C. A. 2000 Small-scale processes and entrainment in a stratocumulus marine boundary layer. J. Atmos. Sci. 57, $567-581$.

Stevens, B. \& Lenschow, D. H. 2001 Observations, experiments, and large eddy simulation. Bull. Am. Met. Soc. 82, 283-294.

Stull, R. B. 1976 The energetics of entrainment across a density interface. J. Atmos. Sci. 33, $1260-1267$.

Sullivan, P. P., Moeng, C.-H., Stevens, B., Lenschow, D. H. \& Mayor, S. D. 1998 Structure of the entrainment zone capping the convective atmospheric boundary layer. J. Atmos. Sci. 55, 3042-3064.

TURNER, J. S. 1968 The influence of molecular diffusivity on turbulent entrainment across a density interface. J. Fluid Mech. 33, 639-656.

Wilson, R., Dalaudier, F. \& Bertin, F. 2005 Estimation of the turbulent fraction in the free atmosphere from MST radar measurements. J. Atmos. Ocean. Technol. 22, 1326-1339.

Winters, K. B. \& D’Asaro, E. A. 1996 Diascalar flux and the rate of fluid mixing. J. Fluid Mech. 317, 179-193.

Winters, K. B., Lombard, P. N., Riley, J. J. \& D’Asaro, E. A. 1995 Available potential energy and mixing in density-stratified fluids. J. Fluid Mech. 289, 115-128.

Xue, M., Droegemeier, K. K. \& Wong, V. 2000 The Advanced Regional Prediction System (ARPS) - a multi-scale non hydrostatic atmospheric simulation and prediction model. Part I: Model dynamics and verification. Met. Atmos. Phys. 75, 161-193.

van Zanten, M. C., Duynkerke, P. G. \& Cuijpers, J. W. M. 1999 Entrainment parametrization in convective boundary layers. J. Atmos. Sci. 56, 813-828.

Zilitinkevich, S. S. 1991 Turbulent Penetrative Convection. Avebury Technical, Aldershot, UK. 